\title{
Research Article Generalized Lower and Upper Approximations in Quantales
}

\author{
Qimei Xiao, ${ }^{1,2}$ and Qingguo Li $^{1}$ \\ ${ }^{1}$ College of Mathematics and Econometrics, Hunan University, Changsha, Hunan 410082, China \\ ${ }^{2}$ School of Mathematics and Computer Science, Changsha University of Science and Technology, \\ Changsha, Hunan 410004, China \\ Correspondence should be addressed to Qingguo Li, liqingguoli@yahoo.com.cn
}

Received 8 August 2011; Revised 21 October 2011; Accepted 30 October 2011

Academic Editor: Jin L. Kuang

Copyright (C) 2012 Q. Xiao and Q. Li. This is an open access article distributed under the Creative Commons Attribution License, which permits unrestricted use, distribution, and reproduction in any medium, provided the original work is properly cited.

\begin{abstract}
We introduce the concepts of set-valued homomorphism and strong set-valued homomorphism of a quantale which are the extended notions of congruence and complete congruence, respectively. The properties of generalized lower and upper approximations, constructed by a set-valued mapping, are discussed.
\end{abstract}

\section{Introduction}

The concept of Rough set was introduced by Pawlak [1] as a mathematical tool for dealing with vagueness or uncertainty. In Pawlak's rough sets, the equivalence classes are the building blocks for the construction of the lower and upper approximations. It soon invoked a natural question concerning a possible connection between rough sets and algebraic systems. Biswas and Nanda [2] introduced the notion of rough subgroups. Kuroki [3] and Qimei [4] introduced the notions of a rough ideal and a rough prime ideal in a semigroup, respectively. Davvaz in [5] introduced the notion of rough subring with respect to an ideal of a ring. Rough modules have been investigated by Davvaz and Mahdavipour [6]. Rasouli and Davvaz studied the roughness in MV-algebra [7]. In [8-12], the roughness of various hyperstructures are discussed. Further, some authors consider the rough set in a fuzzy algebraic system, see [1316]. The concept of quantale was introduced by Mulvey [17] in 1986 with the purpose of studying the spectrum of $C^{*}$-algebra, as well as constructive foundations for quantum mechanics. There are abundant contents in the structure of quantales, because quantale can be regarded as the generalization of frame. Since quantale theory provides a powerful tool in studying noncommutative structures, it has wide applications, especially in studying noncommutative $\mathrm{C}^{*}$-algebra theory, the ideal theory of commutative ring, linear logic, and so on. 
The quantale theory has aroused great interests of many researchers, and a great deal of new ideas and applications of quantale have been proposed in twenty years [18-24].

The majority of studies on rough sets for algebraic structures such as semigroups, groups, rings, and modules have been concentrated on a congruence relation. An equivalence relation is sometimes difficult to be obtained in real-world problems due to the vagueness and incompleteness of human knowledge. From this point of view, Davvaz [25] introduced the concept of set-valued homomorphism for groups. And then, Yamak et al. [26, 27] introduced the concepts of set-valued homomorphism and strong set-valued homomorphism of a ring and a module. In this paper, the concepts of set-valued homomorphism and strong set-valued homomorphism in quantales are introduced. We discuss the properties of generalized lower and upper approximations in quantales.

\section{Preliminaries}

In this section, we give some basic notions and results about quantales and rough set theory (see $[19,22,25,28])$, which will be necessary in the next sections.

Definition 2.1. A quantale is a complete lattice $Q$ with an associative binary operation o satisfying

$$
a \circ\left(\bigvee_{i \in I} b_{i}\right)=\bigvee_{i \in I}\left(a \circ b_{i}\right), \quad\left(\bigvee_{i \in I} a_{i}\right) \circ b=\bigvee_{i \in I}\left(a_{i} \circ b\right)
$$

for all $a, b, a_{i}, b_{i} \in Q(i \in I)$.

An element $e \in Q$ is called a left (right) unit if and only if $e \circ a=a(a \circ e=a), e$ is called a unit if it is both a right and left unit.

A quantale $Q$ is called a commutative quantale if $a \circ b=b \circ a$ for all $a, b \in Q$.

A quantale $Q$ is called an idempotent quantale if $a \circ a=a$ for all $a \in Q$.

A subset $S$ of $Q$ is called a subquantale of $Q$ if it is closed under o and arbitrary sups.

In a quantale $Q$, we denote the top element of $Q$ by 1 and the bottom by 0 . For $A, B \subseteq Q$, we write $A \circ B$ to denote the set $\{a \circ b \mid a \in A, b \in B\}, A \vee B$ to denote $\{a \vee b \mid a \in A, b \in B\}$ and $\bigvee_{i \in I} A_{i}=\left\{\bigvee_{i \in I} a_{i} \mid a_{i} \in A_{i}\right\}$

Definition 2.2. Let $Q$ be a quantale, a subset $\emptyset \neq I \subseteq Q$ is called a left (right) ideal of $Q$ if

(1) $a, b \in I$ implies $a \vee b \in I$,

(2) $a \in I, b \in Q$ and $b \leq a$ imply $b \in I$ for all,

(3) $a \in Q$ and $x \in I$ imply $a \circ x \in I(x \circ a \in I)$.

A subset $I \subseteq Q$ is called an ideal if it is both a left and a right ideal.

Let $X$ be a subset of $Q$, we write $\downarrow X=\{y \in Q \mid y \leq x$ for some $x \in X\}, X$ is a lower set if and only if $X=\downarrow X$. It is obvious that an ideal $I$ is a directed lower set. For every (left, right) ideal $I$ of $Q$, it is easy to see that $0 \in I$.

An ideal of $Q$ is called a prime ideal if $a \circ b \in I$ implies $a \in I$ or $b \in I$ for all $a, b \in Q$.

An ideal $I$ of $Q$ is called a semi-prime ideal if $a \circ a \in I$ implies $a \in I$ for all $a \in Q$.

An ideal $I$ of $Q(I \neq Q)$ is called a primary ideal if for all $a, b \in Q, a \circ b \in I$ and $a \notin I$ imply $b^{n} \in I$ for some $n>0$. $(b^{n}=\underbrace{b \circ b \circ \cdots \circ b}_{n})$. 
Definition 2.3. A nonempty subset $M \subseteq Q$ is called an $m$-system of $Q$, if for all $a, b \in M$, $\downarrow(a \circ 1 \circ b) \cap M \neq \emptyset$.

A nonempty subset $S \subseteq Q$ is called a multiplicative set of $Q$, if $a \circ b \in S$ for all $a, b \in S$.

Every ideal of $Q$ is both an $m$-system and a multiplicative set.

Definition 2.4. Let $Q$ be a quantale, an equivalence relation $\theta$ on $L$ is called a congruence on $Q$ if for all $a, b, c, d, a_{i}, b_{i} \in Q(i \in I)$, we have

(1) $a \theta b, c \theta d \Rightarrow(a \circ c) \theta(b \circ d)$,

(2) $a_{i} \theta b_{i}(i \in I) \Rightarrow\left(\bigvee_{i \in I} a_{i}\right) \theta\left(\bigvee_{i \in I} b_{i}\right)$.

It is obvious that $[a]_{\theta} \circ[b]_{\theta} \subseteq[a \circ b]_{\theta}, \bigvee_{i \in I}\left[a_{i}\right]_{\theta} \subseteq\left[\bigvee_{i \in I} a_{i}\right]_{\theta}$ for all $a, b, a_{i} \in Q(i \in I)$

Definition 2.5. Let $Q$ be a quantale, a congruence $\theta$ on $Q$ is called a complete congruence, if

(1) $[a]_{\theta} \circ[b]_{\theta}=[a \circ b]_{\theta}$ for all $a, b \in Q$,

(2) $\bigvee_{i \in I}\left[a_{i}\right]_{\theta}=\left[\bigvee_{i \in I} a_{i}\right]_{\theta}$ for all $a_{i} \in Q(i \in I)$.

Definition 2.6. Let $\left(Q_{1}, \circ_{1}\right)$ and $\left(Q_{2}, \circ_{2}\right)$ be two quantales. A map $f: Q_{1} \rightarrow Q_{2}$ is said to be a homomorphism if

(1) $f\left(a \circ_{1} b\right)=f(a) \circ_{2} f(b)$ for all $a, b \in Q_{1}$;

(2) $f\left(\bigvee_{i \in I} a_{i}\right)=\bigvee_{i \in I} f\left(a_{i}\right)$ for all $a_{i} \in Q_{1}(i \in I)$.

Definition 2.7. Let $U$ and $W$ be two nonempty universes. Let $T$ be a set-valued mapping given by $T: U \rightarrow P(W)$, where $P(W)$ denotes the set of all subsets of $W$. Then the triple $(U, W, T)$ is referred to as a generalized approximation space. For any set $A \subseteq W$, the generalized lower and upper approximations, $T_{-}(A)$ and $T^{-}(A)$, are defined by

$$
T_{-}(A)=\{x \in U \mid T(x) \subseteq A\}, \quad T^{-}(A)=\{x \in U \mid T(x) \cap A \neq \emptyset\} .
$$

The pair $\left(T_{-}(A), T^{-}(A)\right)$ is referred to as a generalized rough set.

From the definition, the following theorems can be easily derived.

Theorem 2.8. Let $U, W$ be nonempty universes and $T: U \rightarrow P^{*}(W)$ be a set-valued mapping, where $P^{*}(W)$ denotes the set of all nonempty subsets of $W$. If $A \subseteq W$, then $T_{-}(A) \subseteq T^{-}(A)$.

If $W=U$ and $R_{T}=\{(x, y) \mid y \in T(x)\}$ is an equivalence relation on $U$, then the pair $\left(U, R_{T}\right)$ is the Pawlak approximation space. Therefore, a generalized rough set is an extended notion of Pawlak's rough set.

Theorem 2.9. Let $(U, W, T)$ be a generalized approximation space, its lower and upper approximation operators satisfy the following properties. For all $A, B \in P(W)$,

(1) $T_{-}(A)=\left(T^{-}\left(A^{c}\right)\right)^{c}, T^{-}(A)=\left(T_{-}\left(A^{c}\right)\right)^{c}$,

(2) $T_{-}(W)=U, T^{-}(\emptyset)=\emptyset$,

(3) $T_{-}(A \cap B)=T_{-}(A) \cap T_{-}(B), T^{-}(A \cup B)=T^{-}(A) \cup T^{-}(B)$, 
(4) $A \subseteq B \Rightarrow T_{-}(A) \subseteq T_{-}(B), T^{-}(A) \subseteq T^{-}(B)$,

(5) $T_{-}(A \cup B) \supseteq T_{-}(A) \cup T_{-}(B), T^{-}(A \cap B) \subseteq T^{-}(A) \cap T^{-}(B)$,

where $A^{c}$ is the complement of the set $A$.

\section{Generalized Rough Subsets in Quantales}

In this paper, $\left(Q_{1}, \circ_{1}\right)$ and $\left(Q_{2}, \circ_{2}\right)$ are two quantales.

Theorem 3.1. Let $T: Q_{1} \rightarrow P\left(Q_{2}\right)$ be a set-valued mapping and $\emptyset \neq A, B \subseteq Q_{2}$. Then

(1) $T^{-}(A) \cup T^{-}(B) \subseteq T^{-}(A \vee B)$, if $0 \in A \cap B$,

(2) $T^{-}(A) \cup T^{-}(B) \subseteq T^{-}\left(A \circ_{2} B\right)$, if $e \in A \cap B$,

(3) $T_{-}(A) \cap T_{-}(B) \subseteq T_{-}(A \vee B)$,

(4) $T_{-}(A) \cap T_{-}(B) \subseteq T_{-}(A \vee B)$, if $Q_{2}$ is an idempotent quantale,

(5) $T_{-}(A) \cup T_{-}(B) \subseteq T_{-}(A \vee B)$, if $0 \in A \cap B$,

(6) $T_{-}(A) \cup T_{-}(B) \subseteq T_{-}\left(A \circ_{2} B\right)$, if $e \in A \cap B$.

Proof. (1) Suppose that $a \in A$, we have $a=a \vee 0 \in A \vee B$ for $0 \in B$. So $A \subseteq A \vee B$. Similarly, $B \subseteq A \vee B$. So $A \cup B \subseteq A \vee B$. By Theorem 2.9, we have $T^{-}(A) \cup T^{-}(B)=T^{-}(A \cup B) \subseteq T^{-}(A \vee B)$.

(2) Suppose that $a \in A$, we have $a=a \circ_{2} e \in A \circ_{2} B$ for $e \in B$. So $A \subseteq A \circ_{2} B$. Similarly, $B \subseteq A \circ_{2} B$. So $A \cup B \subseteq A \circ_{2} B$. By Theorem 2.9, we have $T^{-}(A) \cup T^{-}(B)=T^{-}(A \cup B) \subseteq$ $\mathrm{T}^{-}\left(\mathrm{A} \mathrm{\circ}_{2} \mathrm{~B}\right)$.

(3) It is obvious that $A \cap B \subseteq A \vee B$. By Theorem 2.9, we have $T_{-}(A) \cap T_{-}(B)=$ $T_{-}(A \cap B) \subseteq T_{-}(A \vee B)$.

(4) Since $Q_{2}$ is an idempotent quantale, we have $A \cap B \subseteq A \circ B$. By Theorem 2.9, we have $T_{-}(A) \cap T_{-}(B)=T_{-}(A \cap B) \subseteq T_{-}\left(A \circ_{2} B\right)$.

(5) and (6) The proofs are similar to (1) and (2), respectively.

Definition 3.2. A set-valued mapping $T: Q_{1} \rightarrow P\left(Q_{2}\right)$ is called a set-valued homomorphism if

(1) $T(a) \circ_{2} T(b) \subseteq T\left(a \circ_{1} b\right)$ for all $a, b \in Q_{1}$ ，

(2) $\bigvee_{i \in I} T\left(a_{i}\right) \subseteq T\left(\bigvee_{i \in I} a_{i}\right)$ for all $a_{i} \in Q_{1}(i \in I)$.

$T$ is called a strong set-valued homomorphism if the equalities in (1), (2) hold.

Example 3.3. (1) Let $\theta$ be a congruence on $Q_{2}$. Then the set-valued mapping $T: Q_{1} \rightarrow P\left(Q_{2}\right)$ defined by $T(x)=[x]_{\theta}$ is a set-valued homomorphism but not necessarily a strong set-valued homomorphism. If $\theta$ is complete, then $T$ is a strong set-valued homomorphism.

(2) Let $f$ be a quantale homomorphism from $Q_{1}$ to $Q_{2}$. Then the set-valued mapping $T: Q_{1} \rightarrow P\left(Q_{2}\right)$ defined by $T(a)=\{f(a)\}$ is a strong set-valued homomorphism.

Theorem 3.4. Let $T: Q_{1} \rightarrow P\left(Q_{2}\right)$ be a set-valued homomorphism and $\emptyset \neq A, B \subseteq Q_{2}$. Then

(1) $T^{-}(A) \vee T^{-}(B) \subseteq T^{-}(A \vee B)$,

(2) $T^{-}(A) \circ_{1} T^{-}(B) \subseteq T^{-}\left(A \circ_{2} B\right)$,

(3) $T^{-}(A) \cap T^{-}(B) \subseteq T^{-}(A \vee B)$,

(4) $T^{-}(A) \cap T^{-}(B) \subseteq T^{-}\left(A \circ_{2} B\right)$, if $Q_{1}$ is an idempotent quantale. 
Proof. (1) Suppose that $c \in T^{-}(A) \vee T^{-}(B)$, there exist $a \in T^{-}(A), b \in T^{-}(B)$ such that $c=a \vee b$. So there exist $x \in A \cap T(a)$ and $y \in B \cap T(b)$. Hence $x \vee y \in A \vee B$ and $x \vee y \in T(a) \vee T(b)$. Since $T$ is a set-valued homomorphism, we have $x \vee y \in T(a \vee b)$. Therefore, $T(a \vee b) \cap(A \vee B) \neq \emptyset$ which implies that $c=a \vee b \in T^{-}(A \vee B)$.

(2) The proof is similar to (1).

(3) Suppose that $x \in T^{-}(A) \cap T^{-}(B)$, there exist $a \in A \cap T(x)$ and $b \in B \cap T(x)$. Since $T$ is a set-valued homomorphism, we have $a \vee b \in T(x) \vee T(x) \subseteq T(x)$. So $a \vee b \in T(x) \cap(A \vee B)$ which implies that $x \in T^{-}(A \vee B)$.

(4) Suppose that $x \in T^{-}(A) \cap T^{-}(B)$, there exist $a \in A \cap T(x)$ and $b \in B \cap T(x)$. Since $T$ is a set-valued homomorphism and $Q_{1}$ is idempotent, we have $a \vee b \in T(x) \circ_{2} T(x) \subseteq$ $T\left(x \circ_{1} x\right)=T(x)$. So $a \vee b \in T(x) \cap(A \vee B)$ which implies that $x \in T^{-}\left(A \circ_{2} B\right)$.

Theorem 3.5. Let $T: Q_{1} \rightarrow P\left(Q_{2}\right)$ be a strong set-valued homomorphism and $\emptyset \neq A, B \subseteq Q_{2}$. Then

(1) $T_{-}(A) \vee T_{-}(B) \subseteq T_{-}(A \vee B)$,

(2) $T_{-}(A) \circ_{1} T_{-}(B) \subseteq T_{-}\left(A \circ_{2} B\right)$.

Proof. (1) Suppose that $c \in T_{-}(A) \vee T_{-}(B)$, there exist $a \in T_{-}(A), b \in T_{-}(B)$ such that $c=a \vee b$. Hence $T(a) \subseteq A$ and $T(b) \subseteq B$. Since $T$ is a strong set-valued homomorphism, we have $T(a \vee b)=T(a) \vee T(b) \subseteq A \vee B$ which implies that $c=a \vee b \in T_{-}(A \vee B)$.

(2) The proof is similar to (1).

\section{Generalized Rough Ideals in Quantales}

Theorem 4.1. Let $T: Q_{1} \rightarrow P\left(Q_{2}\right)$ be a set-valued mapping. If I and J are, respectively, a right and a left ideal of $Q_{2}$, then

(1) $T^{-}\left(I \circ_{2} J\right) \subseteq T^{-}(I) \cap T^{-}(J)$,

(2) $T_{-}\left(I \circ_{2} J\right) \subseteq T_{-}(I) \cap T_{-}(J)$,

(3) $T^{-}(I \wedge J) \subseteq T^{-}(I) \cap T^{-}(J)$,

(4) $T_{-}(I) \wedge T_{-}(J)=T_{-}(I \wedge J)$,

(5) $T^{-}(I) \cup T^{-}(J) \subseteq T^{-}(I \vee J)$,

(6) $T_{-}(I) \cup T_{-}(J) \subseteq T_{-}(I \vee J)$.

If $Q_{1}$ is an idempotent quantale and $T$ is a set-valued homomorphism, then the equalities in (1)-(3) hold.

Proof. Since $I$ and $J$ are, respectively, a right and a left ideal of $Q_{2}$, we have $I \circ_{2} J \subseteq I \cap J$, $I \wedge J=I \cap J$ and $o \in I \cap J$. By Theorem 2.9, we get the conclusion (1)-(4). By Theorem 3.1, we get (5) and (6).

If $Q_{1}$ is idempotent, we first show that $T^{-}(I) \cap T^{-}(J) \subseteq T^{-}\left(I \circ_{2} J\right)$. Suppose that $x \in T^{-}(I) \cap T^{-}(J)$, there exist $y \in I, z \in J$ such that $y, z \in T(x)$. So, $y \circ_{2} z \in I \circ_{2} J$ and $y \circ_{2} z \in T(x) \circ_{2} T(x)$. Since T is a set-valued homomorphism and $Q_{1}$ is idempotent, we have $y \circ_{2} z \in T\left(x \circ_{1} x\right)=T(x)$. Therefore, $x \in T^{-}\left(I \circ_{2} J\right)$. So the equality in (1) holds. Since $Q_{1}$ is idempotent, we have $I \cap J \subseteq I \circ_{2} J$. So $I \cap J=I \circ_{2} J=I \cap J$. By Theorem 2.9, we get $T_{-}\left(I \circ_{2} J\right)=T_{-}(I \cap J)=T_{-}(I) \cap T_{-}(J)$. Since the equality in (1) holds, we have $T^{-}(I \wedge J)=$ $T^{-}\left(I \circ_{2} J\right)=T^{-}(I) \cap T^{-}(J)$. 
Lemma 4.2. Let $T: Q_{1} \rightarrow P\left(Q_{2}\right)$ be a set-valued homomorphism. If $A$ is a lower set of $Q_{2}$, then $T_{-}(A)$ is a lower set of $Q_{1}$.

Proof. Suppose $x \leq y \in T_{-}(A)$, then $T(y) \subseteq A$. Let $z \in T(x), a \in T(y)$, we have $z \vee a \in T(x) \vee$ $T(y) \subseteq T(x \vee y)=T(y) \subseteq A$. Since $A$ is a lower set, we have $z \in A$. Therefore, $T(x) \subseteq A$ which implies that $x \in T_{-}(A)$.

Lemma 4.3. Let $T: Q_{1} \rightarrow P\left(Q_{2}\right)$ be a strong set-valued homomorphism. If $A$ is a lower set of $Q_{2}$, then $T^{-}(A)$ is a lower set of $Q_{1}$.

Proof. Suppose that $x \leq y \in T^{-}(A)$, there exists $z \in T(y) \cap A$. Since $T$ is a strong set-valued homomorphism, we have $T(x) \vee T(y)=T(x \vee y)=T(y)$. So there exist $a \in T(x), b \in T(y)$ such that $z=a \vee b$. Since $A$ is a lower set, we have $a \in A$. Thus $T(x) \cap A \neq \emptyset$ which follows that $x \in T^{-}(A)$.

Lemma 4.4. Let $T: Q_{1} \rightarrow P\left(Q_{2}\right)$ be a set-valued homomorphism and $A$ a nonempty subset of $Q_{2}$. If $A$ is closed under arbitrary (resp., finite) sups, then $T^{-}(A)$ is closed under arbitrary (resp., finite) sups.

Proof. Let $B \subseteq T^{-}(A)$. For each $b \in B$, we have $b \in T^{-}(A)$, then there exist $x_{b} \in T(b) \cap A$. Since $T$ is a set-valued homomorphism, we have $\bigvee_{b \in B} x_{b} \in \bigvee_{b \in B} T(b) \subseteq T(\bigvee B)$. And we have $\bigvee_{b \in B} x_{b} \in A$ for $\mathrm{A}$ is closed under arbitrary sups. So $T(\bigvee B) \cap A \neq \emptyset$ which implies that $\bigvee B \in$ $T^{-}(A)$.

Lemma 4.5. Let $T: Q_{1} \rightarrow P\left(Q_{2}\right)$ be a strong set-valued homomorphism and $A$ a nonempty subset of $Q_{2}$. If $A$ is closed under arbitrary (resp., finite) sups, then $T_{-}(A)$ is closed under arbitrary (resp., finite) sups.

Proof. Let $B \subseteq T_{-}(A)$. For each $b \in B$, we have $T(b) \subseteq A$. Since $T$ is a strong set-valued homomorphism, we have $T(\bigvee B)=\bigvee T(B)$. Suppose $z \in T(\bigvee B)=\bigvee T(B)$, there exist $x_{b} \in T(b) \subseteq$ $A(b \in B)$ such that $z=\bigvee_{b \in B} x_{b}$. Since $A$ is closed under arbitrary sups, we have $z=\bigvee_{b \in B} x_{b} \in$ $A$. So $T(\bigvee B) \subseteq A$ which implies that $\bigvee B \in T_{-}(A)$.

Theorem 4.6. Let $T: Q_{1} \rightarrow P\left(Q_{2}\right)$ be a set-valued homomorphism. If $A$ is a subquantale of $Q_{2}$, then $T^{-}(A)$ is a subquantale of $Q_{1}$.

Proof. Since $T$ is a set-valued homomorphism and $A$ is a subquantale, by Theorems 3.4 and 2.9, we have $T^{-}(A) \circ_{1} T^{-}(A) \subseteq T^{-}\left(A \circ_{2} A\right) \subseteq T^{-}(A)$. So, $T^{-}(A)$ is closed under $\circ_{1}$.

Since $A$ is closed under arbitrary sups, by Lemma 4.4 , we get $T^{-}(A)$ is closed under arbitrary sups.

Theorem 4.7. Let $T: Q_{1} \rightarrow P\left(Q_{2}\right)$ be a strong set-valued homomorphism. If $A$ is a subquantale of $Q_{2}$, then $T_{-}(A)$ is a subquantale of $Q_{1}$.

Proof. The proof is similar to Theorem 4.6.

Theorem 4.8. Let $T: Q_{1} \rightarrow P^{*}\left(Q_{2}\right)$ be a strong set-valued homomorphism and I a right (left) ideal of $Q_{2}$. Then $T^{-}(I)$ is, if it is nonempty, a right (left) ideal of $Q_{1}$.

Proof. Suppose that $a, b \in T^{-}(I)$. Since $I$ is closed under finite sups, by Lemma 4.4, we have $T^{-}(I)$ is closed under finite sups. So $a \vee b \in T^{-}(I)$. 
Table 1

\begin{tabular}{llll}
\hline$\circ_{1}$ & 0 & $a$ & 1 \\
\hline 0 & 0 & $a$ & 1 \\
$a$ & 0 & $a$ & 1 \\
1 & 0 & $a$ & 1 \\
\hline
\end{tabular}

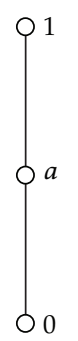

Figure 1

Since $I$ is a lower set, by Lemma 4.3, we have $T^{-}(I)$ is a lower set.

Suppose $a \in Q_{1}, x \in T^{-}(I)$, there exists $y \in I \cap T(x)$. Since $I$ is a right ideal of $Q_{2}$, we have $y \circ_{2} b \in I$ for each $b \in T(a) \subseteq Q_{2}$. So $y \circ_{2} b \in T(x) \circ_{2} T(a) \subseteq T\left(x \circ_{1} a\right)$. Therefore, $T\left(x \circ_{1} a\right) \cap I \neq \emptyset$ which implies that $x \circ_{1} a \in T^{-}(I)$.

Theorem 4.9. Let $T: Q_{1} \rightarrow P\left(Q_{2}\right)$ be a strong set-valued homomorphism and I a right (left) ideal of $Q_{2}$. Then $T_{-}(I)$ is, if it is nonempty, a right (left) ideal of $Q_{1}$.

Proof. Suppose $a, b \in T_{-}(I)$. Since $I$ is closed under finite sups, by Lemma 4.5, we have $T_{-}(I)$ is closed under finite sups. So, $a \vee b \in T_{-}(I)$.

Since $I$ is a lower set, by Lemma 4.2 , we have $T_{-}(I)$ is a lower set.

Suppose $a \in Q_{1}, x \in T_{-}(I)$, we have $T(x) \subseteq I$. Let $y \in T\left(x \circ_{1} a\right)$. Since $T$ is a strong set-valued homomorphism, we have $y \in T(x) \circ_{2} T(a)$, then there exist $y_{1} \in T(x) \subseteq I, y_{2} \in$ $T(a)$ such that $y=y_{1} \circ_{2} y_{2}$. Since $I$ is a right ideal, we have $y=y_{1} o_{2} y_{2} \in I$. Therefore, $T\left(x \circ_{1} a\right) \subseteq I$ which implies that $x \circ_{1} a \in T_{-}(I)$.

Definition 4.10. A subset $A \subseteq Q_{2}$ is called a generalized rough ideal (subquantale) of $Q_{1}$ if $T_{-}(A)$ and $T^{-}(A)$ are ideals (subquantales) of $Q_{1}$.

The following corollary follows from Theorems 4.6-4.9.

Corollary 4.11. Let $T: Q_{1} \rightarrow P\left(Q_{2}\right)$ be a strong set-valued homomorphism and I an ideal (a subquantale) of $Q_{2}$. If $T_{-}(I)$ and $T^{-}(I)$ are nonempty, then $I$ is a generalized rough ideal (subquantale) of $Q_{1}$.

From the above, we know that an ideal is a generalized rough ideal with respect to a strong set-valued homomorphism. The following example shows that the converse does not hold in general.

Example 4.12. Let $Q_{1}=\{0, a, 1\}$ and $Q_{2}=\left\{0^{\prime}, b, c, 1^{\prime}\right\}$ be quantales shown in Figures 1 and 2 and Tables 1 and 2.

Let $T: Q_{1} \rightarrow P\left(Q_{2}\right)$ be a strong set-valued homomorphism as defined by $T(0)=\left\{0^{\prime}\right\}$, $T(a)=\{b, c\}, T(1)=\left\{1^{\prime}\right\}$. Let $A=\left\{0^{\prime}, b, c\right\} \subseteq Q_{2}, B=\{b, c\} \subseteq Q_{2}$, then $T_{-}(A)=\{0, a\}=T^{-}(A)$ 
Table 2

\begin{tabular}{ccccc}
\hline $\mathrm{o}_{2}$ & $0^{\prime}$ & $b$ & $c$ & $1^{\prime}$ \\
\hline $0^{\prime}$ & $0^{\prime}$ & $b$ & $c$ & $1^{\prime}$ \\
$b$ & $0^{\prime}$ & $b$ & $c$ & $1^{\prime}$ \\
$c$ & $0^{\prime}$ & $b$ & $c$ & $1^{\prime}$ \\
$1^{\prime}$ & $0^{\prime}$ & $b$ & $c$ & $1^{\prime}$ \\
\hline
\end{tabular}

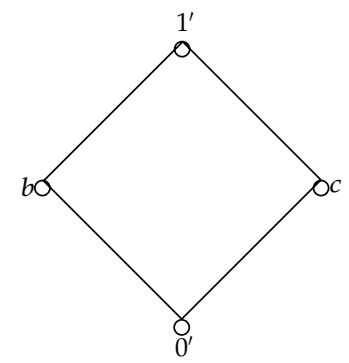

Figure 2

and $T_{-}(B)=\{a\}=T^{-}(B)$. It is obvious that $A$ is a generalized rough ideal of $Q_{1}$ but $A$ is not an ideal of $Q_{2}$ and $B$ is a generalized rough subquantale of $Q_{1}$ but $B$ is not a subquantale of $Q_{2}$.

Theorem 4.13. Let $T: Q_{1} \rightarrow P\left(Q_{2}\right)$ be a strong set-valued homomorphism and I a prime ideal of $Q_{2}$. Then $T^{-}(I)$ is, if it is nonempty, a prime ideal of $Q_{1}$.

Proof. By Theorem 4.8, we get $T^{-}(I)$ is an ideal of $Q_{1}$.

Let $a \circ_{1} b \in T^{-}(I)$, there exists $x \in I \cap T\left(a \circ_{1} b\right)$. Since $T$ is a strong set-valued homomorphism, we have $x \in T(a) \circ_{2} T(b)$, then there exist $y \in T(a), z \in T(b)$ such that $x=$ $y \circ_{2} z \in I$. Since $I$ is a prime ideal of $Q_{2}$, we have $y \in I$ or $z \in I$. So $T(a) \cap I \neq \emptyset$ or $T(b) \cap I \neq \emptyset$ which implies that $a \in T^{-}(I)$ or $b \in T^{-}(I)$.

Theorem 4.14. Let $T: Q_{1} \rightarrow P\left(Q_{2}\right)$ be a strong set-valued homomorphism and I a prime ideal of $Q_{2}$. Then $T_{-}(I)$ is, if it is nonempty, a prime ideal of $Q_{1}$.

Proof. By Theorem 4.9, we get $T_{-}(I)$ is an ideal of $Q_{1}$.

Let $a \circ_{1} b \in T_{-}(I)$, we have $T\left(a \circ_{1} b\right) \subseteq I$. Since $T$ is a strong set-valued homomorphism, we have $T(a) \circ_{2} T(b) \subseteq I$. We assume that $a \notin T_{-}(I)$, then $T(a) \nsubseteq I$, there exists $x \in T(a)$ but $x \notin I$. If $y \in T(b)$, then $x \circ_{2} y \in T(a) \circ_{2} T(b) \subseteq I$. Since $I$ is a prime ideal of $Q_{2}$, we have $y \in I$. Therefore, $T(b) \subseteq I$ which implies that $b \in T_{-}(I)$.

$Q_{1}$.

We call $I \subseteq Q_{2}$ is a generalized rough prime ideal of $Q_{1}$ if $T_{-}(I)$ and $T^{-}(I)$ are ideals of

Theorem 4.15. Let $T: Q_{1} \rightarrow P\left(Q_{2}\right)$ be a strong set-valued homomorphism and I a semiprime ideal of $Q_{2}$. Then $T_{-}(I)$ is, if it is nonempty, a semiprime ideal of $Q_{1}$. 
Proof. By Theorem 4.9, we get $T_{-}(I)$ is an ideal of $Q_{1}$.

Suppose that $a \circ_{1} a \in T_{-}(I)$, we have $T\left(a \circ_{1} a\right) \subseteq I$. Let $x \in T(a)$, we have $x \circ_{2} x \in$ $T(a) \circ_{2} T(a) \subseteq T\left(a \circ_{1} a\right) \subseteq I$. Since $I$ is a semi-prime ideal, we have $x \in I$. So $T(a) \subseteq I$ which implies that $a \in T_{-}(I)$.

Theorem 4.16. Let $T: Q_{1} \rightarrow P\left(Q_{2}\right)$ be a strong set-valued homomorphism and I a primary ideal of $Q_{2}$. Then $T^{-}(I)$ is, if $T^{-}(I) \neq \emptyset$ and $T^{-}(I) \neq Q_{1}$, a primary ideal of $Q_{1}$.

Proof. By Theorem 4.8, we get $T^{-}(I)$ is an ideal of $Q_{1}$.

Suppose that $a, b \in Q, a \circ_{1} b \in T^{-}(I)$ and $a \notin T^{-}(I)$, there exists $x \in I \cap T\left(a \circ_{1} b\right)$. Since $T$ is a strong set-valued homomorphism, we have $x \in T(a) \circ_{2} T(b)$, there exist $y \in T(a)$, $z \in T(b)$ such that $x=y \circ_{2} z \in I$. Since $a \notin T^{-}(I)$, we get $y \notin I$. Since $I$ is a primary ideal, we have $z^{n} \in I$ for some $n>0$ Since $T$ is a strong set-valued homomorphism, we have $z^{n} \in T\left(b^{n}\right)$. So $T\left(b^{n}\right) \cap I \neq \emptyset$ which implies that $b^{n} \in T^{-}(I)$.

Theorem 4.17. Let $T: Q_{1} \rightarrow P\left(Q_{2}\right)$ be a strong set-valued homomorphism and I a primary ideal of $Q_{2}$. Let $Q_{2}$ be a commutative quantale and $T(x)$ a finite set for each $x \in Q_{1}$. Then $T_{-}(I)$ is, if $T_{-}(I) \neq \emptyset$ and $T_{-}(I) \neq Q_{1}$, a primary ideal of $Q_{1}$.

Proof. By Theorem 4.9, we get $T_{-}(I)$ is an ideal of $Q_{1}$.

Suppose $a, b \in Q, a \circ_{1} b \in T_{-}(I)$ and $a \notin T_{-}(I)$, then $T\left(a \circ_{1} b\right) \subseteq I$ and $T(a) \nsubseteq I$. So there exists $x \in T(a)$ with $x \notin I$. We assume that $T(b)=\left\{y_{1}, y_{2}, \ldots, y_{m}\right\}$, then $x \circ_{2} y_{i} \in$ $T(a) \circ_{2} T(b) \subseteq T\left(a \circ_{1} b\right) \subseteq I(i=1,2, \ldots, m)$. Since $I$ is a primary ideal, there exists $y_{i}^{n_{i}} \in I$ for some $n_{i}>0(i=1,2, \ldots, m)$. Let $n=\max \left\{n_{i} \mid i=1,2, \ldots, m\right\}$. Since $I$ is an ideal, we have $y_{i}^{l} \in I(l \geq n)$. Let $z \in T\left(b^{m} b^{n}\right)=T(b)^{m n}$. Since $Q_{2}$ is commutative, there exists $\left\{i_{1}, i_{2}, \ldots, i_{s}\right\} \subseteq$ $\{1,2, \ldots, m\}$ such that $z=y_{i_{1}}^{t_{1}} \circ_{2} y_{i_{2}}^{t_{2}} \circ_{2} \cdots \circ_{2} y_{i_{s}}^{t_{s}}$ with $t_{1}+t_{2}+\cdots+t_{s}=m n$, where $t_{j}>0(j=1$, $2, \ldots, s)$. Assume that $t_{j}<n,(j=1,2, \ldots, s)$, then $t_{1}+t_{2}+\cdots+t_{s}<s n \leq m n$. It contradicts with $t_{1}+t_{2}+\cdots+t_{s}=m n$. Therefore, there is $1 \leq k \leq s$ such that $t_{k} \geq n,(j=1,2, \ldots, s)$, we have $y_{i_{k}}^{t_{k}} \in I$. Since $I$ is an ideal, we get $z \in I$. Hence $T\left(b^{m n}\right) \subseteq I$ which implies that $b^{m n} \in T_{-}(I)$.

Theorem 4.18. Let $T: Q_{1} \rightarrow P\left(Q_{2}\right)$ be a set-valued homomorphism. If $S$ is a multiplicative set of $Q_{2}$, then $T^{-}(S)$ is, if it is nonempty, a multiplicative of $Q_{1}$. If $T$ is a strong set-valued homomorphism, then $T_{-}(S)$ is, if it is nonempty, a multiplicative of $Q_{1}$.

Proof. Suppose that $a, b \in T^{-}(S)$, there exist $x \in T(a) \cap S, y \in T(b) \cap S$. Hence $x \circ y \in T(a) \circ_{2}$ $T(b) \subseteq T\left(a \circ_{1} b\right)$. Since $S$ is a multiplicative set, we have $x \circ_{2} y \in S$. Therefore, $x \circ_{2} y \in T\left(a \circ_{1} b\right) \cap S$ which implies that $a \circ_{1} b \in T^{-}(S)$.

Suppose $a, b \in T_{-}(S)$, then $T(a) \subseteq S, T(b) \subseteq S$. Let $x \in T\left(a \circ_{1} b\right)$. Since $T$ is a strong set-valued homomorphism, we have $x \in T(a) \circ_{2} T(b)$. There exist $y \in T(a) \subseteq S, z \in T(b) \subseteq S$ such that $x=y \circ_{2} z$. Since $S$ is a multiplicative set, we have $x=y \circ_{2} z \in S$. So $T\left(a \circ_{1} b\right) \subseteq S$ which implies that $a \circ_{1} b \in T_{-}(S)$.

We call $A \subseteq Q_{2}$ a generalized rough multiplicative set (m-system) of $Q_{1}$, if $T_{-}(A)$, $T^{-}(A)$ are multiplicative sets ( $m$-systems) of $Q_{1}$.

Theorem 4.19. Let $T: Q_{1} \rightarrow P^{*}\left(Q_{2}\right)$ be a set-valued homomorphism. If $Q_{1}$ is commutative and $A \subseteq$ $Q_{2}$ with $1 \notin T^{-}(A)$, then the following statements are equivalent:

(1) $A$ is a generalized rough prime ideal of $Q_{1}$,

(2) $A$ is a generalized rough ideal and $A^{c}$ is a generalized rough multiplicative set of $Q_{1}$,

(3) $A$ is a generalized rough ideal and $A^{c}$ is a generalized rough m-system of $Q_{1}$. 
Proof. (1) $\Rightarrow(2)$ : Since $A$ is a generalized rough prime ideal of $Q_{1}$, we get $A$ is a generalized rough ideal of $Q_{1}$ and $T_{-}(A), T^{-}(A)$ are prime ideals of $Q_{1}$. Now we show that $T^{-}\left(A^{c}\right)$ is a multiplicative set. Let $a, b \in T^{-}\left(A^{c}\right)$. By Theorem 2.9(1), we have $a, b \in\left(T_{-}(A)\right)^{c}$. Since $T_{-}(A)$ is a prime ideal, we have $a \circ_{1} b \notin T_{-}(A)$ which implies that $a \circ_{1} b \in\left(T_{-}(A)\right)^{c}=T^{-}\left(A^{c}\right)$. So $T^{-}\left(A^{c}\right)$ is a multiplicative set. Similarly, $T_{-}\left(A^{c}\right)$ is a multiplicative set.

$(2) \Rightarrow(3)$ : Let $a, b \in T^{-}\left(A^{c}\right)$. Since $1 \notin T^{-}(A)$ and $T(1) \neq \emptyset$, we have $1 \in T^{-}\left(A^{c}\right)$. Since $T^{-}\left(A^{c}\right)$ is a multiplicative set, we have $a \circ_{1} 1 \circ_{1} b \in T^{-}\left(A^{c}\right)$. So $\downarrow\left(a \circ_{1} 1 \circ_{1} b\right) \cap T^{-}\left(A^{c}\right) \neq \emptyset$. Therefore, $T^{-}\left(A^{c}\right)$ is an $m$-system. Similarly, we have $T_{-}\left(A^{c}\right)$ is a $m$-system.

(3) $\Rightarrow(1)$ : Let $a \circ_{1} b \in T^{-}(A)$. Suppose $a \notin T^{-}(A)$ and $b \notin T^{-}(A)$, then $a, b \in\left(T^{-}(A)\right)^{c}=$ $T_{-}\left(A^{c}\right)$. Since $T_{-}\left(A^{c}\right)$ is an $m$-system, we have $\downarrow\left(a \circ_{1} 1 \circ_{1} b\right) \cap T_{-}\left(A^{c}\right) \neq \emptyset$. Thus $\downarrow\left(a \circ_{1}\right.$ $\left.1 \circ_{1} b\right) \cap\left(T^{-}(A)\right)^{c} \neq \emptyset$. Since $T^{-}(A)$ is an ideal and $Q_{1}$ is commutative, we have $a \circ_{1} 1 \circ_{1} b \in$ $T^{-}(A)$ and $\downarrow\left(a \circ_{1} 1 \circ_{1} b\right) \subseteq T^{-}(A)$. It contradicts with $\downarrow\left(a \circ_{1} 1 \circ_{1} b\right) \cap\left(T^{-}(A)\right)^{c} \neq \emptyset$. So, $a \in T^{-}(A)$ or $b \in T^{-}(A)$. Therefore, $T^{-}(A)$ is a prime ideal. Similarly, we have $T_{-}(A)$ as a prime ideal.

\section{Conclusion}

The Pawlak rough sets on the algebraic sets such as semigroups, groups, rings, modules, and lattices were mainly studied by a congruence relation. However, the generalized Pawlak rough set was defined for two universes and proposed on generalized binary relations. Can we extended congruence relations to two universes for algebraic sets? Therefore, Davvaz [25] introduced the concept of set-valued homomorphism for groups which is a generalization of the concept of congruence. In this paper, the concepts of set-valued homomorphism and strong set-valued homomorphism of quantales are introduced. We construct generalized lower and upper approximations by means of a set-valued mapping and discuss the properties of them. We obtain that the concept of generalized rough ideal (subquantale) is the extended notion of ideal (subquantale). Many results in [29] are the special case of the results in this paper. It is an interesting research topic of rough set, we will further study it in the future.

\section{Acknowledgments}

The authors are grateful to the reviewers for their valuable suggestions to improve the paper. This work was supported by the National Science Foundation of China (no. 11071061).

\section{References}

[1] Z. Pawlak, "Rough sets," International Journal of Computer and Information Sciences, vol. 11, no. 5, pp. 341-356, 1982.

[2] R. Biswas and S. Nanda, "Rough groups and rough subgroups," Bulletin of the Polish Academy of Sciences Mathmatics, vol. 42, no. 3, pp. 251-254, 1994.

[3] N. Kuroki, "Rough ideals in semigroups," Information Sciences, vol. 100, no. 1-4, pp. 139-163, 1997.

[4] X. Qimei and Z. Zhenliang, "Rough prime ideals and rough fuzzy prime ideals in semigroups," Information Sciences, vol. 176, no. 6, pp. 725-733, 2006.

[5] B. Davvaz, "Roughness in rings," Information Sciences, vol. 164, no. 1-4, pp. 147-163, 2004.

[6] B. Davvaz and M. Mahdavipour, "Roughness in modules," Information Sciences, vol. 176, no. 24, pp. 3658-3674, 2006. 
[7] S. Rasouli and B. Davvaz, "Roughness in MV-algebras," Information Sciences, vol. 180, no. 5, pp. 737$747,2010$.

[8] B. Davvaz, "A new view of approximations in Hv-groups," Soft Computing, vol. 10, no. 11, pp. 10431046, 2006.

[9] B. Davvaz, "Approximations in hyperrings," Journal of Multiple-Valued Logic and Soft Computing, vol. 15, no. 5-6, pp. 471-488, 2009.

[10] O. Kazanc1, S. Yamak, and B. Davvaz, "The lower and upper approximations in a quotient hypermodule with respect to fuzzy sets," Information Sciences, vol. 178, no. 10, pp. 2349-2359, 2008.

[11] V. Leoreanu-Fotea and B. Davvaz, "Roughness in n-ary hypergroups," Information Sciences, vol. 178, no. 21, pp. 4114-4124, 2008.

[12] V. L. Fotea, "The lower and upper approximations in a hypergroup," Information Sciences, vol. 178, no. 18, pp. 3605-3615, 2008.

[13] B. Davvaz, "Roughness based on fuzzy ideals," Information Sciences, vol. 176, no. 16, pp. 2417-2437, 2006.

[14] O. Kazanc1 and B. Davvaz, "On the structure of rough prime (primary) ideals and rough fuzzy prime (primary) ideals in commutative rings," Information Sciences, vol. 178, no. 5, pp. 1343-1354, 2008.

[15] N. Kuroki and P. P. Wang, "The lower and upper approximations in a fuzzy group," Information Sciences, vol. 90, no. 1-4, pp. 203-220, 1996.

[16] J. N. Mordeson, "Rough set theory applied to (fuzzy) ideal theory," Fuzzy Sets and Systems, vol. 121, no. 2, pp. 315-324, 2001.

[17] C. J. Mulvey, “\&," Rendiconti del Circolo Matematico di Palermo II, supplement 12, pp. 99-104, 1986.

[18] J. Picado, "The quantale of Galois connections," Algebra Universalis, vol. 52, no. 4, pp. 527-540, 2004.

[19] K. I. Rosenthal, Quantales and Their Applications, vol. 234 of Pitman Research Notes in Mathematics Series, Longman Scientific \& Technical, Harlow, UK, 1990.

[20] J. Rosický, “Characterizing spatial quantales," Algebra Universalis, vol. 34, no. 2, pp. 175-178, 1995.

[21] H. Shengwei and Z. Bin, "The quantic conuclei on quantales," Algebra Universalis, vol. 61, no. 1, pp. 97-114, 2009.

[22] W. Shunqin and Z. Bin, "Ideals of quantales," Journal of Shaanxi Normal University, vol. 31, no. 4, pp. 7-10, 2003.

[23] W. Shunqin and Z. Bin, "Prime ideals and weakly prime ideals of quantales," Fuzzy Systems and Mathematics, vol. 19, no. 1, pp. 78-81, 2005.

[24] D. N. Yetter, "Quantales and (noncommutative) linear logic," The Journal of Symbolic Logic, vol. 55, no. 1, pp. 41-64, 1990.

[25] B. Davvaz, "A short note on algebraic T-rough sets," Information Sciences, vol. 178, no. 16, pp. 32473252, 2008.

[26] S. Yamak, O. Kazanc1, and B. Davvaz, "Generalized lower and upper approximations in a ring," Information Sciences, vol. 180, no. 9, pp. 1759-1768, 2010.

[27] S. Yamak, O. Kazanc1, and B. Davvaz, "Approximations in a module by using set-valued homomorphism," International Journal of Computer Mathematics, vol. 88, pp. 2901-2914, 2011.

[28] Z. Wenxiu and W. Weizhi, Theory and Method of Roughness, Science Press, Beijing, China, 2001.

[29] Y. Lingyun, Algebraic and Topological Study on Formal Concept Analysis and Rough Set Theory [D], Yangzhou University, 2010. 


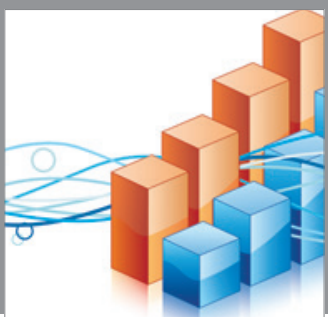

Advances in

Operations Research

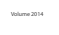

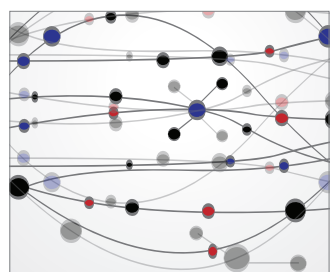

\section{The Scientific} World Journal
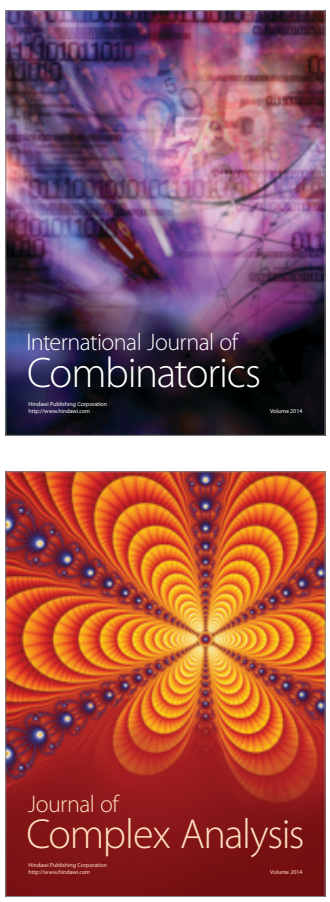

International Journal of

Mathematics and

Mathematical

Sciences
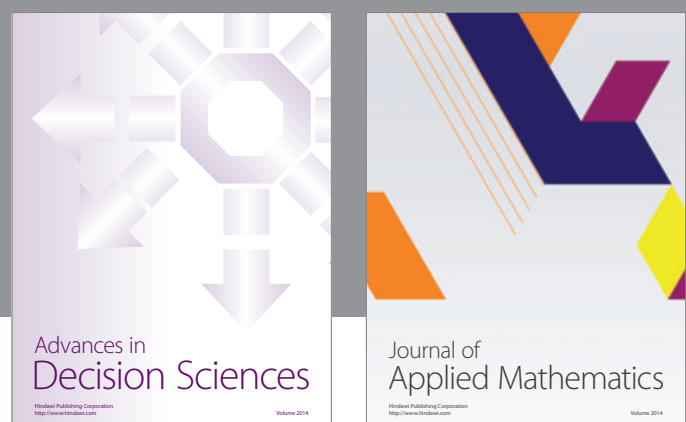

Journal of

Applied Mathematics
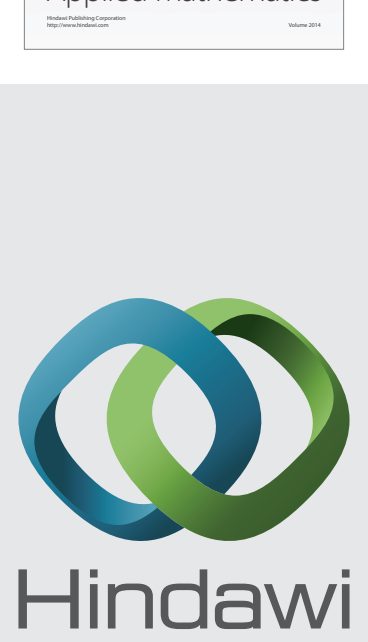

Submit your manuscripts at http://www.hindawi.com
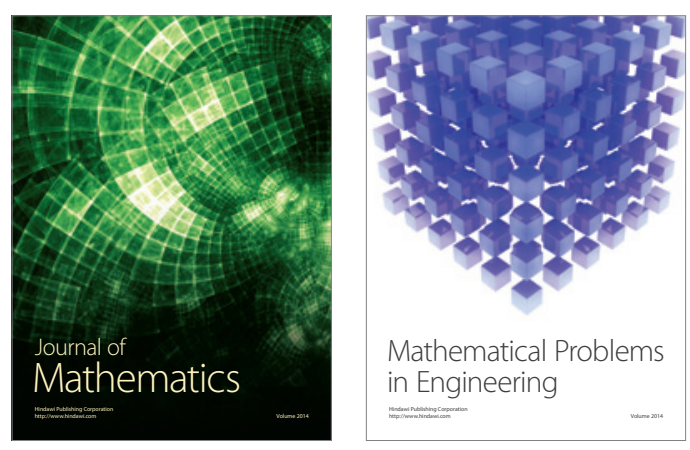

Mathematical Problems in Engineering
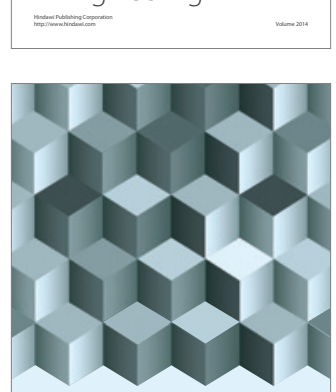

Journal of

Function Spaces
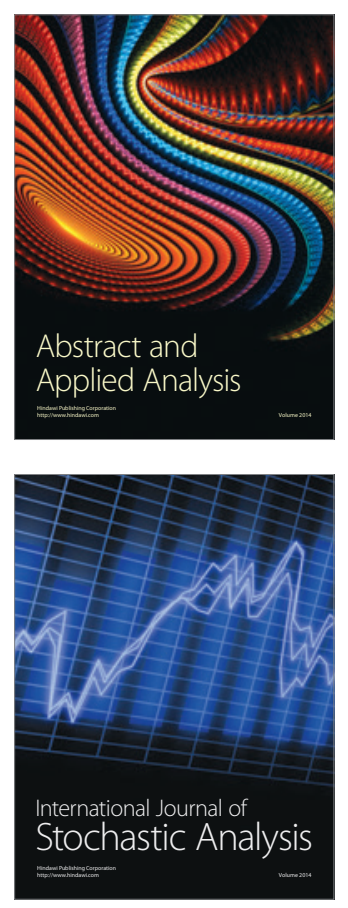

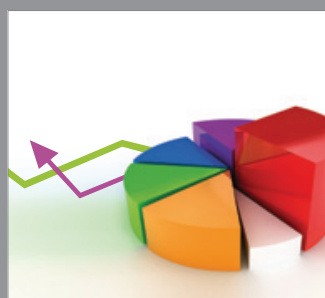

ournal of

Probability and Statistics

Promensencen
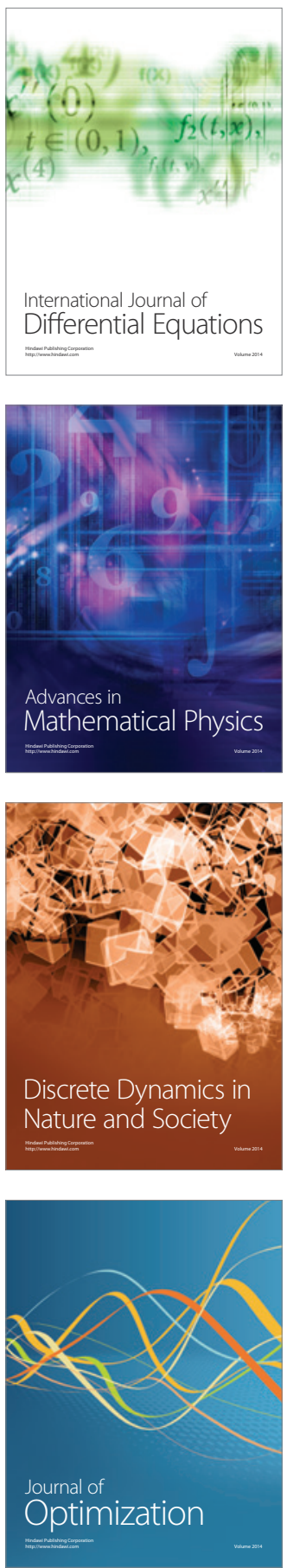